Conclusions Understanding the common pediatric diagnoses presenting at EDs can facilitate clinical guidelines implementation, resource allocation and staff training. By focusing efforts on emergent (P1) diagnoses, general EDs can enhance their preparedness and quality of pediatric emergency care to critically ill patients.

\section{ANTENATALLY DIAGNOSED RENAL ANOMALIES AND THE SIX-MONTH POST-NATAL OUTCOME OF RENAL PELVIC DILATATION AT A DISTRICT GENERAL HOSPITAL IN SOUTH EAST ENGLAND}

Cinderella Menezes, Bindu Radha, Soraya Shirzad, Ahmed Abdelgadir. UK

\subsection{6/bmjpo-2021-RCPCH.177}

Background Congenital anomalies of kidney and renal tract (CAKUT) constitute $20-30 \%$ of all anomalies identified perinatally. CAKUT play causative role in $30-50 \%$ chronic kidney disease requiring renal replacement treatment in children. Majority of these anomalies are not life threatening. It is important to diagnose and initiate treatment early to prevent progression to end stage renal disease

Objectives

- To collect data on antenatally diagnosed renal anomalies and their demographics at our centre.

- To study the six-month outcome of foetuses identified with renal pelvic dilatation (RPD).

- To identify areas of improvement and implement changes.

Methods We identified cases from records of prenatal multidisciplinary team (MDT) meetings from January to December 2019. Data was retrospectively collected from our hospital database, maternal and neonatal records, laboratory and radiology results and clinic letters.

Results 46 foetuses with renal anomalies were discussed in the MDT, 11 discharged antenatally, 1 moved home. 34 were included in the study $(n=34)$.

Foetuses with bilateral(B/L) RPD:

9 foetuses were diagnosed with $\mathrm{B} / \mathrm{L}$ RPD, 8 were male and 1 female.

5 needed urgent renal ultrasound - 100\% done within 2448 hrs, 4 needed routine scans, all done between 1-4 weeks.

8 were started on antibiotic prophylaxis.

First postnatal scan showed $\mathrm{B} / \mathrm{L} \mathrm{RPD}$ in $2, \mathrm{U} / \mathrm{L} \mathrm{RPD}$ in 4 and normal in 3 .

Further investigations done included MCUG in 5, MAG3 scans in 3 and DMSA in 1.

\begin{tabular}{ll} 
Abstract 321 Table 1 & Antenatally diagnosed anomalies \\
\hline Antenatally diagnosed anomalies & Number \\
\hline Bilateral RPD & 9 \\
Unilateral RPD only & 6 \\
Unilateral RPD with calyceal/ureteric involvement & 6 \\
Unilateral Multi cystic dysplastic kidney (MCKD) & 4 \\
Duplex kidney & 4 \\
Unilateral renal agenesis & 2 \\
Unilateral MCKD + other side duplex & 1 \\
Echogenic kidneys & 1 \\
Supra renal cyst & 1 \\
\hline
\end{tabular}

At 6 months, antibiotic prophylaxis was stopped in 5 and discharged from care, 1 had planned follow up with paediatrician at 1 year and 3 had ongoing care under urology.

Foetuses with unilateral(U/L) RPD

12 foetuses with $\mathrm{U} / \mathrm{L} \mathrm{RPD} \pm$ Calyceal/ureteral involvement.

10 were male, 2 females.

11 had routine renal US. First ultrasound findings were normal in 5, unilateral RPD in 3, duplex kidney in 1, hydronephrosis in 2 .

1 had urgent scan on day 1 , which revealed mild calyceal fullness in absence of RPD.

10 were started on trimethoprim prophylaxis. At six months, prophylaxis was stopped in 7 , continued in 1 and not specified in 2 .

Conclusions In $\mathrm{B} / \mathrm{L} \mathrm{RPD}$, targets for prophylaxis were met in $96.4 \%$, and in 100\% patients for urgent scans. No cases on posterior urethral valves were diagnosed in the study period.

In U/L RPD, targets for scan were met in $100 \%$ and 91.6\% for antibiotic prophylaxis.

To improve compliance with prophylactic antibiotics, our Trust patient information leaflet on RPD is modified to add information on antibiotic prophylaxis and more stringent measures are taken to update the neonatal team about antenatal diagnoses.

\section{ASSESSING THE IMPACT OF BLENDED OUTREACH AND TELEMEDICINE PAEDIATRIC NEUROLOGY SPECIALIST SERVICES IN MYANMAR}

Kyaw Linn, Marcus Wootton, Aye Mya Min Aye, Chaw Su Hlaing, Ayemu Saan, Khine Mi Mi Ko. Myanmar

\subsection{6/bmjpo-2021-RCPCH.178}

Background Myanmar has an improving health picture with significant reductions in neonatal and paediatric mortality. However, there remain significant regional variations in specialist expertise and care. Regarding the paediatric neurology services, there are only 11 paediatric neurologists for a paediatric population of about 14 million children $(26 \%$ of the total population). Children in Myanmar's rural communities, $70 \%$ of the total population, live in conditions which both drive the neurological burden and limit access to effective treatment.

Recognising the burden and barriers to specialist services, we present evidence from an expanding programme of blended outreach and telemedicine with the overall aim of increasing the number of children in remote areas who gain access to specialist neurological assessment and treatment.

Objectives This study aimed to assess the scope and impact of those outreach and telemedicine paediatric neurology specialist services.

Methods The outreach clinics were of a two-stream model with face-to-face consultations by up to three paediatric neurologists from Yangon. This service was supplemented using telemedicine clinics and mobile instant messaging applications for follow up and any emergency problems between individual outreach clinics.

Whilst most children were seen on an outpatient basis, the visiting specialist also saw admitted children with neurological problems during outreach clinic visits providing an expert opinion. Continued medical education activities were also conducted for local doctors and nurses. Data including patient 
numbers, different neurological conditions, cost-effectiveness, and patient satisfaction responses were analysed.

Results We started visits to regional hospitals in 2017 and 2018 before upscaling in 2019, to provide quarterly visits to 7 regional and district hospitals who had previously had no access to any paediatric neurology service provision aside from tertiary referral. Altogether, 1327 children with different neurological problems were seen in 2019. This included 712 children (54\%) with epilepsy, 155 children (12\%) with cerebral palsy and 100 children (8\%) with other developmental problems.

The costs of facilitating the clinics were compared to the equivalent cost of the patients travelling to a tertiary centre for the same level of specialist assessment and treatment. Percentage of cost-saving was from $81 \%$ to $98 \%$ depending on the distance between the regional hospital and Yangon where the tertiary centre is located.

We conducted a small survey of parents attending the clinic. Respondents felt that the outreach clinics provided a more accessible point of care. Many highlighted the lifting of a significant financial burden, particularly in low-income households. The reduction of financial cost is felt particularly in cases such as epilepsy which require multiple clinic visits and ongoing specialist care. Many comments have also identified the quality of specialist care in the new model, comparable to a tertiary hospital.

Conclusions Paediatric neurology outreach and virtual clinics can provide a model for other specialities across many fields of healthcare, especially in countries with good internet, but poor transport. Interval telemedicine services also provided a continuation of care. To achieve specialist care at greater scale new approaches, such as the blended outreach and telemedicine structure described here, should be actively developed and evaluated.

\section{IMMUNOSUPPRESSIVE THERAPIES IN CHILDREN WITH BIOPSY-PROVEN IGA VASCULITIS NEPHRITIS: A TERTIARY CENTRE EXPERIENCE}

Hon Lam Matthew Lee, Eugene Yu-hin Chan. Hong Kong

\subsection{6/bmjpo-2021-RCPCH.179}

Background IgA Vasculitis Nephritis (IgAVN) can lead to severe presentation including nephrotic syndrome. Data pertaining to the treatment outcomes of IgAVN with persistent moderate or nephrotic range proteinuria in children are, however, limited.

Objectives The aim of this study is to determine the response to immunosuppressive therapies in this patient population.

Methods We conducted a retrospective review on all children presenting with IgAV before 18 years between January 2009 and December 2019 in the Paediatric Nephrology Centre in Hong Kong. Patients with biopsy-proven IgAVN developing persistent moderate or severe nephrotic-range proteinuria despite ACE-inhibitor (ACEi), and followed for 24 months or more were included. Patient demographics, clinical and laboratory data, therapies received, and treatment outcomes were evaluated.

Results Of the 177 patients with IgAV, 42 children developed proteinuria. 21 Chinese patients (76\% boy) had persistent proteinuria despite ACEi and kidney biopsy confirmed IgAVN at a median age of 8.5 years (IQR 5.8-11.2). At baseline, 3
(14\%), 14 (66\%), 3 (14\%) and 1 (4\%) patients had moderate proteinuria, nephrotic-range proteinuria, nephrotic syndrome and nephritic-nephrotic syndrome, respectively. All patients had normal kidney function, except one child with an estimated GFR of $31 \mathrm{ml} / \mathrm{min} / 1.73 \mathrm{~m}^{2}$. Median urine protein to urine creatinine ratio (UPCR) was $4.4 \mathrm{mg} / \mathrm{mg}$ (IQR 2.4-9.0) and serum albumin was $32 \mathrm{~g} / \mathrm{L}$ (IQR 28-33.5). Histological findings were classified according to International Study of Kidney Diseases in Children (ISKDC): Class II $(\mathrm{n}=5,24 \%)$, Class IIIa $(n=9,42 \%)$, Class IIIb $(n=6,29 \%)$, Class IV $(n=1$, $5 \%)$.

All patients received corticosteroid at a median time of 33 days (IQR 12-52) since kidney involvement. Whereas 7 children (33\%) with severe disease received monthly intravenous cyclophosphamide as induction therapy, 12 patients $(57 \%)$ and 2 patients (10\%) received calcineurin inhibitors and azathioprine, respectively. The maintenance therapy consisted of corticosteroid and one additional immunosuppression, including calcineurin inhibitors $(n=16,76 \%)$, azathioprine $(n=4,19 \%)$ and mycophenolate mofetil $(n=1,5 \%)$.

Over a median follow-up period of 3.6 years (IQR 2.85.6), 18 patients (86\%) attained complete remission at a median of 139.5 days (IQR 102-225) since immunosuppressants initiation. The other 3 patients achieved partial remission. Three patients (14\%) relapsed in 7.5 months (IQR 1.216.2) following complete remission but resolved promptly with treatments. At last follow-up, all patients had normal kidney function and the median UPCR was $0.11 \mathrm{mg} / \mathrm{mg}$ (IQR 0.10-0.16).

Conclusions Immunosuppressive therapies were associated with favourable renal outcomes in children with biopsy-proven IgAVN presented with persistent moderate or nephrotic range proteinuria despite ACEi. Further studies are required to determine the optimal treatments in this patient population.

\section{9 HOW EFFECTIVE ARE WE COMMUNICATING WITH OUR PAEDIATRIC ONCOLOGY PATIENTS?}

Olajumoke Osofisan, Thilani Ranasinghe. UK

\subsection{6/bmjpo-2021-RCPCH. 180}

Background Given the sensitive and intricate nature of oncological cases, especially in paediatric age group, it is important to have specific and strategic ways of discussing and communicating diagnosis and treatment plan. Especially being aware that to achieve the trust and faith of our patients and relatives in paediatric oncology, effective communication is key.

Thus, emphasis of both verbal and non-verbal communication with oncology patient cannot be over emphasised.

Objectives Our survey highlighted an assessment of how our communication of diagnosis and care has been with emphasis on striving for improvement. Examples of communications asked about includes, how well the diagnosis was discussed the first time, and how much of the information given was fully understood in the easiest possible manner.

Also, emphasis was placed on regular care given with each admission and how well our team communicated our line of management provided at each step of the way.

There were other areas covered in the questionnaire including support from community nurses, tertiary centres, play specialists and medications given. 